\title{
CAPÍTULO 66
}

\section{O COMPARTILHAMENTO DE NOTÍCIAS FALSAS EM MEIO À PANDEMIA E O IMPACTO NA PROMOÇÃO DE ESTRATÉGIAS DE SAÚDE}

DOI 10.4322/978-65-995353-2-1.c66

\author{
Amanda Cristina da Silva ${ }^{1}$, Sabrina Francisca Felix ${ }^{2}$, \\ Yasmin Yuri Okamoto Beltrão ${ }^{3}$, Natália Augusto Benedetti ${ }^{4}$. \\ Faculdade Marechal Rondon- FMR (Mahdesilva0@gmail.com) ${ }^{1}$ \\ Faculdade Marechal Rondon- FMR (Sabrinafelix105@gmail.com) ${ }^{2}$ \\ Faculdade Marechal Rondon-FMR (minbeltrao@ gmail.com) ${ }^{3}$ \\ Enfermagem da Faculdade Marechal Rondon-FMR(natalia.benedetti@ fmr.pro.br) ${ }^{4}$.
} Mestre em enfermagem pela Universidade Estadual Paulista- UNESP/ Docente do curso de

\section{RESUMO}

OBJETIVO: Promover por meio de uma revisão bibliográfica uma reflexão acerca de como o compartilhamento de notícias sem veracidade científica durante a pandemia Covid-19 atrapalha os avanços dos profissionais de saúde que buscam promover estratégias de saúde e garantir um atendimento de qualidade. MÉTODO: Para a fundamentação desse estudo foram realizadas pesquisas nas bases de dados MEDLINE e SciELO utilizando como descritores as palavras 'Covid-19', ' 'Compartilhamento de informação'" e 'Educação em saúde' de acordo com a classificação dos Descritores em Ciências de Saúde (DESC). Para a organização do processo de identificação das evidências encontradas e maior qualidade dessa publicação, aderiu-se ao modelo de fluxograma de seleção de estudos PRISMA. RESULTADO: Foram identificados 32 estudos com potencial para elegibilidade (MedLine/PubMed=17; Scielo=12; Outras fontes $=1$. Dos 32 estudos identificados, 6 foram excluídos por duplicidade detectados pelo Endnote Basic, permanecendo 26 artigos para a etapa de leitura. Após a etapa de leitura foram excluídos 22 artigos por não estabelecerem uma relação concisa entre o fenômeno de notícias falsas e as consequências para o indivíduo e a saúde pública durante a pandemia do coronavírus, sendo incluídos para compor o trabalho 5 artigos onde; 4 foram identificados por meio de bancos de dados e registros e 1 identificados por meio de outras fontes. CONCLUSÃO: Considera-se uma opção para o combate ao compartilhamento de informações falsas, a alfabetização digital e em saúde. Incentivada pelas próprias plataformas digitais que são os principais propagadores de informações errôneas sobre o coronavírus e por meio delas, compartilhar conteúdo verificado com viés científico, dando voz aos verdadeiros conhecedores, os profissionais de saúde.

Palavras-chave: Covid-19; Compartilhamento de informação; Educação em saúde.

Área Temática: Ciências da Saúde; 
E-mail do autor principal: Mahdesilva0@ gmail.com 1 INTRODUÇÃO

A nova doença coronavírus (COVID-19) foi declarada uma pandemia pela Organização Mundial da Saúde (OMS) em 11 de março de 2020 (OMS, 2020). Esta pandemia e as medidas de saúde pública implementada para reduzi-la mudaram profundamente o estilo de vida das pessoas e é considerada uma ameaça para o bem-estar físico e mental. A natureza imprevisível da doença, a perda de liberdades pessoais, mensagens conflitantes das autoridades, mudanças repentinas nos planos para o futuro imediato ou a preocupação com a própria saúde e bem-estar e de parentes são exemplos de fontes de estresse associado a esses surtos e pandemias (Huremović, 2019).

Durante a pandemia, a mídia digital surgiu como a primeira opção de informação, seguida por telejornais, redes sociais e aplicativos de mensagens instantâneas (Masip et al.,2020). Para que uma notícia falsa tenha sucesso, basta que o assunto chame a atenção. A crise da saúde é uma questão de interesse máximo, pelo que era previsível que se tornasse um terreno fértil para mentiras e trotes, um ambiente propício ao desenvolvimento de notícias falsas. Além disso, é uma questão altamente carregada emocionalmente, portanto, nos momentos em que a comunicação política coloca o emocional antes do racional, o sucesso das notícias falsas é garantido(Del rey,2007).

Na era da informação atual, com abundância de conteúdo disponível gratuitamente, há um problema crescente com as pessoas formando crenças errôneas e muitas vezes prejudiciais após encontrarem "notícias falsas" online (Ali e Levine, 2008). Nesse ambiente, com alto índice de informação disponível no meio digital, encontra-se o problema causado pela alta produção de notícias falsas, mais conhecidas pelo termo 'Fake News', e, consequentemente, o aumento das desinformações nos meios digitais (CARVALHO e MATEUS,2018).

Hoje, praticamente qualquer pessoa é capaz de criar uma notícia no meio digital. Os cidadãos deixaram de ser meros consumidores de conteúdo para se tornarem "'prosumer' (termo originalmente criado por Alvin Toffler em seu livro de 1980 "'The Third Wav"'( A quinta onda) que significa um consumidor que atua também como produtor (Berrocal; Dominguez; Redondo,2014). A desinformação sobre o COVID-19 proliferou amplamente nas redes sociais, desde a venda de falsas "curas", como gargarejo com limão ou água salgada, beber álcool para proteção contra COVID-19, injetar alvejante em você mesmo, as falsas teorias da conspiração de que o vírus foi criado em um laboratório em Wuhan ou que a rede celular $5 \mathrm{G}$ está causando ou agravando os sintomas de COVID-19 (OMS,2020).

\section{E - book Tripé do Ensino Superior: Ensino,} Pesquisa e Extensão 
Na luta contra o coronavírus, é preciso ter como guia as evidencias. Informações errôneas sobre a COVID-19 já foi motivo de ataques a profissionais de enfermagem no Brasil, essas notícias inverídicas podem além de incentivar o comportamento que facilita a transmissão do coronavírus, sobrecarrega ainda mais os hospitais e quem trabalha neles(Conselho Federal de Enfermagem- COFEN,2020).

À medida que a pandemia cresce em todo o mundo, não estamos apenas lidando com o vírus e sua capacidade de sobrecarregar as instituições de saúde, mas também com uma onda de desinformação que está custando vidas humanas e impactando negativamente aqueles que trabalham pela saúde e bem-estar da população. Essa situação também esclareceu a necessidade de educar a população e a mídia e expôs preconceitos contra a comunidade médica(Merchant e Asch,2018).

Essa crise é também uma oportunidade para pensar como os profissionais de saúde, podem contribuir para o enfrentamento da infodemia com ações cotidianas, como compartilhar conteúdo verificado em nossas redes sociais pessoais e profissionais, ouvir e esclarecer dúvidas de pacientes e educar amigos e familiares (Alvarez e Tijerina,2020)

Nessa perspectiva, o objetivo deste trabalho é promover por meio de uma revisão bibliográfica uma reflexão acerca de como o compartilhamento de notícias sem veracidade científica durante a pandemia COVID-19 atrapalha os avanços dos profissionais de saúde que buscam promover estratégias de saúde e garantir um atendimento de qualidade em meio aos desafios de uma pandemia.

\section{MÉTODO}

A fim de atender o objetivo proposto, utilizou-se como método a revisão integrativa da literatura, visto que o propósito desse método de pesquisa é obter um profundo entendimento de um determinado fenômeno baseando-se em estudos anteriores (Broome,2000 citado por; Mendes; Silveira; Galvão,2008).

Para fundamentar a reflexão proposta no objetivo deste estudo foram realizadas pesquisas nas bases de dados; Medical Literature Analysis and Retrieval System OnlineMEDLINE( acesso via PubMed) e Scientific Electronic Library Online (SciELO). Os descritores utilizados para direcionar a pesquisa foram 'Covid-19', ' Compartilhamento de informação" e "Educação em saúde" de acordo com a classificação dos Descritores em Ciências de Saúde (DESC). Buscas também foram feitas nas referências bibliográficas nos artigos encontrados. O ano delimitado para a inclusão no estudo foi a partir do ano de 2020.

E - book Tripé do Ensino Superior: Ensino, Pesquisa e Extensão 
A consulta à base de dados foi realizada nos meses de junho e julho de 2021 e os idiomas de redação dos artigos aceitos foram em português e inglês.

Como critérios de inclusão, foram utilizados somente aqueles artigos que relacionassem o fenômeno social das notícias falsas com a pandemia causada pelo coronavírus e que dessa relação ocasionasse o comprometimento das intervenções baseadas em evidencias com consequências a curto e longo prazo ao indivíduo e a saúde pública.

Para a organização do processo de identificação das evidências encontradas e maior qualidade dessa publicação, aderiu-se ao modelo de fluxograma de seleção de estudos PRISMA.

\section{RESULTADOS E DISCUSSÃO}

Conforme a figura 1 , foram identificados 32 estudos com potencial para elegibilidade (MedLine/PubMed=17; Scielo=12; Outras fontes=1). Os estudos categorizados como ' Outras fontes" são provenientes de buscas realizadas nas referências bibliográficas dos artigos elegíveis para compor este estudo. Dos 32 estudos identificados, 6 foram excluídos por duplicidade detectados pelo Endnote Basic, permanecendo 26 artigos para a etapa de leitura.

Após a etapa de leitura foram excluídos 22 artigos por não estabelecerem uma relação concisa entre o fenômeno de notícias falsas e as consequências para o indivíduo e a saúde pública durante a pandemia do coronavírus, sendo incluídos para compor o trabalho 5 artigos onde; 4 foram identificados por meio de bancos de dados e registros e 1 identificados por meio de outras fontes.

Todos os estudos foram publicados a partir do ano de 2020, em português, inglês e espanhol, não se limitando apenas aqueles específicos à área da saúde. Em seguida conforme mostra a figura 2 os registros foram organizados em uma tabela de acordo com o título, autor(s), idioma, base de dados e endereço eletrônico. 
Figura 1 Diagrama de fluxo PRISMA 2020, para novas revisões sistemáticas que incluíram pesquisas em bancos de dados, registros e outras fontes.

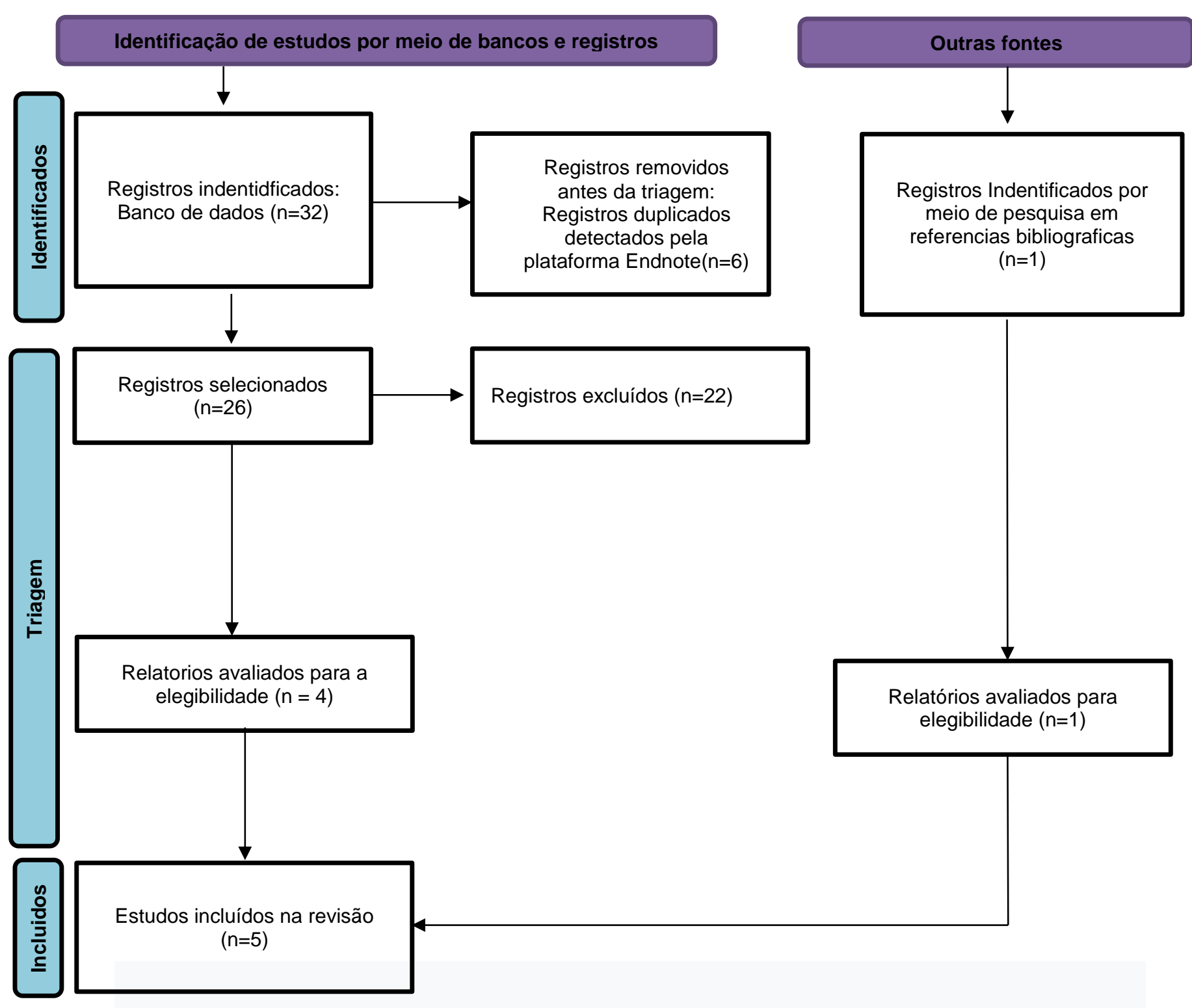

Fonte:http://www.prisma-statement.org/

Figura 2 Registros incluídos na revisão segundo título, autor(s), idioma, base de dados e endereço eletrônico.

\begin{tabular}{|c|c|c|c|c|}
\hline TÍTULO & AUTOR(S) & IDIOMA & $\begin{array}{l}\text { BASE DE } \\
\text { DADOS }\end{array}$ & ENDEREÇO ELETRÔNICO \\
\hline $\begin{array}{l}\text { Fato ou Fake? Uma } \\
\text { análise da } \\
\text { desinformação } \\
\text { frente à pandemia } \\
\text { da Covid-19 no } \\
\text { Brasil. }\end{array}$ & $\begin{array}{l}\text { Cláudia Pereira } \\
\text { Galhardi,Neyson } \\
\text { Pinheiro } \\
\text { Freire,Maria Cecília } \\
\text { de Souza Minayo, } \\
\text { Maria Clara e } \\
\text { Marques Fagundes }\end{array}$ & Português & SciELO & $\begin{array}{l}\text { https://www.scielo.br/j/csc/a/XnfpYRR } \\
\text { 45Z4nXskC3PTnp8z/?lang=pt }\end{array}$ \\
\hline
\end{tabular}




\begin{tabular}{|c|c|c|c|c|}
\hline $\begin{array}{l}\text { Análise de fake } \\
\text { news veiculadas } \\
\text { durante a } \\
\text { pandemia de } \\
\text { COVID-19 no } \\
\text { Brasil. }\end{array}$ & $\begin{array}{l}\text { Thainá do } \\
\text { Nascimento de } \\
\text { Barcelos,Luíza } \\
\text { Nepomuceno } \\
\text { Muniz,Deborah } \\
\text { Marinho Dantas, } \\
\text { Dorival Fagundes } \\
\text { Cotrim Junior, João } \\
\text { Roberto Cavalcante e } \\
\text { Eduardo Faerstein }\end{array}$ & Português & SciElo & $\begin{array}{l}\text { https://www.scielosp.org/article/rpsp/20 } \\
\text { 21.v45/e65/ }\end{array}$ \\
\hline $\begin{array}{l}\text { Fighting fake } \\
\text { news in the } \\
\text { COVID-19 era: } \\
\text { policy insights } \\
\text { from an } \\
\text { equilibrium } \\
\text { model. }\end{array}$ & $\begin{array}{l}\text { Kris Hartley e Minh } \\
\text { Khuong Vu }\end{array}$ & Inglês & $\begin{array}{l}\text { MEDLINE/PubM } \\
\text { ed }\end{array}$ & $\begin{array}{l}\text { https://www.ncbi.nlm.nih.gov/pmc/artic } \\
\text { les/PMC7479406/ }\end{array}$ \\
\hline $\begin{array}{l}\text { Fake news in } \\
\text { COVID-19: A } \\
\text { perspective. }\end{array}$ & $\begin{array}{l}\text { Diego Carrion- } \\
\text { Alvarez e Perla X. } \\
\text { Tijerina-Salina }\end{array}$ & Inglês & $\begin{array}{l}\text { MEDLINE/Pubm } \\
\text { ed }\end{array}$ & $\begin{array}{l}\text { https://www.ncbi.nlm.nih.gov/pmc/artic } \\
\text { les/PMC7722992/ }\end{array}$ \\
\hline $\begin{array}{l}\text { Impact of } \\
\text { Rumors and } \\
\text { Misinformation } \\
\text { on COVID-19 in } \\
\text { Social Media. }\end{array}$ & $\begin{array}{l}\text { Samia Tasnim, Md } \\
\text { Mahbub } \\
\text { Hossain e Hoimonty } \\
\text { Mazumder }\end{array}$ & Inglês & $\begin{array}{l}\text { MEDLINE/PubM } \\
\text { ed }\end{array}$ & $\begin{array}{l}\text { https://www.ncbi.nlm.nih.gov/pmc/artic } \\
\text { les/PMC7280809/ }\end{array}$ \\
\hline
\end{tabular}

Fonte: Amanda Cristina da Silva, Sabrina Francisca Felix, Yasmin Yuri Okamoto Beltrão, Natalia Augusto Benedetti, 2021.

Dos artigos analisados o de Galhardi CM, et al.,2020 destaca as principais fake News compartilhadas em aplicativos de mensagens e redes sociais e aponta que o fenômeno do compartilhamento de notícias falsas contribuiu para o descrédito da ciência e das instituições de saúde publica enfraquecendo a adesão da população aos cuidados necessários para evitar a contaminação pelo coronavírus. $\mathrm{O}$ artigo ainda considera que o cidadão confira a veracidade do conteúdo a ser compartilhado e que o debate público é uma forma de aumentar a consciência social.

Barcelos TN, et al.,2021, também reconhece que o as fake News comprometem os cuidados comprovados pela ciência, manipulando os valores individuais levando as pessoas a acreditarem no que melhor convém, tudo devido ao estado de medo e incertezas decorrentes de uma pandemia. Essas informações falsas que estão carregadas de potencial para influenciar comportamentos que concordem com interesses políticos, sociais e econômicos são extremamente prejudiciais à instalação de medidas de controle e cura da infecção causada pelo vírus SARs-Cov-2.

\section{E - book Tripé do Ensino Superior: Ensino, Pesquisa e Extensão}


Sobre os efeitos negativos das notícias falsas Kris Hartley e Minh Khuong Vu, 2020, reconhecem que no contexto de uma pandemia até um parcela mínima da população poderia comprometer os esforços para a recuperação de uma crise. Que até mesmo líderes políticos são vulneráveis às notícias falsas. O artigo coloca como exemplo a promoção do ex-presidente dos Estados Unidos da América_Donald Trump no uso de medicamento antimalárico hidroxicloroquina ao qual a comunidade científica, na época, não possuía evidências sobre eficácia. Segundo o artigo isso “ Ilustra os riscos existenciais apresentados por forças políticas construídas na redefinição ou rejeição de fatos científicos"'. De modo geral, o estudo alicerça uma base para explorar as narrativas falsas produzidas em um contexto de crise global, como a da infecção pelo coronavírus.

O artigo de Alvarez D.C e Tijerina-Salina P.X também fala sobre a influência das figuras públicas que são modelos de comportamento e que durante a pandemia contradisseram os conselhos médicos impactando negativamente a população. Os autores levantaram como medida de intervenção não só a educação em saúde, como também a educação sobre o uso de mídias sociais. E que os profissionais de saúde podem contribuir com o compartilhamento consciente de informação, ou seja, conteúdo cientificamente confiável utilizando suas redes sociais para o compartilhamento de conteúdo verificado e, ouvindo e esclarecendo as dúvidas dos pacientes, assim como, educar familiares e amigos.

O artigo de Tasnim S, et al., 2020 fala em como a desinformação sobre o Covid compromete práticas saudáveis e promove comportamentos errôneos levando a resultados ruins para a saúde física e mental do indivíduo. O artigo cita alguns resultados dessa desinformação, como, os vários casos de overdose de cloroquina na Nigéria e o suicido de um pai de três filhos na índia que se suicidou após a contaminação pelo coronavírus. A desinformação generalizada segundo Tasnim S, et ak., 2020 reduz a legitimidade de novas descobertas científicas o que pode ter impacto prolongado na nutrição da saúde pública e na saúde psicossocial. Como medida para conter a disseminação da desinformação é levantado à responsabilidade das mídias sociais em eliminar fraudes e promover a divulgação de conteúdo cientificamente confiável, e que os profissionais devem estar equipados com os resultados das pesquisas recentes para que se tenha facilidade na comunicação entre o paciente e o provedor de saúde. $\mathrm{O}$ artigo ainda levanta a telemedicina como potencial fornecedor de cuidados baseados em evidências e serviço informativo. 


\section{CONCLUSÃO}

A partir dos dados analisados e do objetivo proposto neste trabalho, evidencia-se que o compartilhamento de notícias falsas se mostrou extremamente prejudicial para estabelecer medidas de saúde para impedir a disseminação do vírus e o estabelecimento de hábitos de vida saúdavéis, buscando a promoção e a qualidade de vida em meio à pandemia de Covid-19. Considera-se uma opção para o combate ao compartilhamento de informações falsas, a alfabetização digital e em saúde. Incentivada pelas próprias plataformas digitais que são os principais propagadores de informações errôneas sobre o coronavírus e por meio delas, compartilhar conteúdo verificado com viés científico, dando voz aos verdadeiros conhecedores, os profissionais de saúde.

\section{REFERÊNCIAS}

Ali, M., \& Levine, T. The language of truthful and deceptive denials and confessions. Communication Reports,2008, 21(2), 82-91.

Alvarez D, C and Tijerina-Salina , P. Fake news in COVID-19: A perspective. Health Promot Perspect. 2020; 10(4): 290-291.

Barcelos, Thainá do Nascimento et al. Análise de fake news veiculadas durante a pandemia de COVID-19 no Brasil. Revista Panamericana de Salud Pública [online]. v. 45, e65. Disponível em: <https://doi.org/10.26633/RPSP.2021.65>. ISSN 1680-5348.

https://doi.org/10.26633/RPSP.2021.65.

Berrocal Gonzalo, S .; Campos Domínguez, E .; Redondo García, M. Prosumidores mediáticos en la comunicación política: El «politainment» en YouTube. Comun. Rev. Científica Iberoam. Comun. Educ. 2014 , 43 , 65-72.

Carrion-Alvarez D, Tijerina-Salina PX. Fake news in COVID-19: A perspective. Health Promot Perspect. 2020;10(4):290-291. Published 2020 Nov 7. doi:10.34172/hpp.2020.44

Carvalho, M. F.C; Mateus, C. A. fake news e desinformação no meio digital: análise da produção científica sobre o tema na área de ciência da informação. Encontro regional de 
estudantes de biblioteconomia, documentação, gestão e ciência da informação das regiões, sudeste, centro-oeste e sul. Belo horizonte- MG 2018.

Conselho federal de enfermagem COFEN adere a carta dos profissionais de saúde contra Fake News,2020.

Del Rey Morató, J. Comunicación Política, Internet y Campañas Electorales: De la Teledemocracia a la Ciberdemocr @ cia ; Tecnos: Barcelona, Espanha, 2007; ISBN 978-84-3094506-1.

Galhardi, Cláudia Pereira et al. Fato ou Fake? Uma análise da desinformação frente à pandemia da Covid-19 no Brasil. Ciência \& Saúde Coletiva [online]. 2020, v. 25, suppl 2, pp. 4201-4210. Disponível em: <https://doi.org/10.1590/1413-812320202510.2.28922020>. Epub 30 Set 2020. ISSN 1678-4561. https://doi.org/10.1590/1413-812320202510.2.28922020.

Hartley K, Vu MK. Fighting fake news in the COVID-19 era: policy insights from an equilibrium model [published online ahead of print, 2020 Sep 9]. Policy Sci. 2020;1-24. doi:10.1007/s11077-020-09405-z

Huremović;Distanciamento social, quarentena e isolamento. Psychiatry of Pandemics,2019 pp. 85-94.

Masip, p.; Aran-Ramspott, S; Ruiz Caballero, C.; Suau, j.; Almenar, E,; Puertas-Graell, D. Consumo Informativo y cobertura mediática durante el confinamento por el Covid-19; Sobreinformacion, sesgo ideológico y sensacionalismo. Inf Prof.2020.

Merchant RM, Asch DA. Protecting the value of medical science in the age of social media and “fake news” JAMA. 2018;320(23):2415-6. doi: 10.1001/jama.2018.18416.

Organização Mundial da Saúde. Conselhos sobre doença coronavírus (COVID19)paraopúblico:CaçadoresdeMitos, 2020. Disponívelonlineem: https://www.who.int/emergencies/diseases/novel-coronavirus-2019/advice-for-public/mythbusters 
Tasnim S, Hossain MM, Mazumder H. Impact of Rumors and Misinformation on COVID-19 in Social Media. J Prev Med Public Health. 2020;53(3):171-174. doi:10.3961/jpmph.20.094 